\title{
Skeletal muscle quality assessed from echo intensity is associated with muscle strength of middle- aged and elderly persons.
}

\section{$\operatorname{AUTHOR}(S):$}

Fukumoto, Yoshihiro; Ikezoe, Tome; Yamada, Yosuke; Tsukagoshi, Rui; Nakamura, Masatoshi; Mori, Natsuko; Kimura, Misaka; Ichihashi, Noriaki

\section{CITATION:}

Fukumoto, Yoshihiro ...[et al]. Skeletal muscle quality assessed from echo intensity is associated with muscle strength of middle-aged and elderly persons.. European journal of applied physiology 2012, 112(4): 1519-1525

\section{ISSUE DATE:}

2012-04

URL:

http://hdl.handle.net/2433/156269

\section{RIGHT:}

The final publication is available at www.springerlink.com; This is not the published version. Please cite only the published version.; この論文 は出版社版でありません。引用の際には出版社版をご確認ご利用くだ さい。 
Skeletal muscle quality assessed from echo intensity is associated with muscle strength of middle-aged and elderly persons

Yoshihiro Fukumoto (RPT, MS) ${ }^{1,2}$, Tome Ikezoe (RPT, PhD) $)^{1}$, Yosuke Yamada $(\mathrm{PhD})^{3}$, Rui Tsukagoshi (RPT, MS) ${ }^{1,2}$, Masatoshi Nakamura (RPT, MS) ${ }^{1,2}$, Natsuko Mori (RPT, MS) ${ }^{4}$, Misaka Kimura $(\mathrm{PhD})^{3}$, and Noriaki Ichihashi $(\mathrm{RPT}, \mathrm{PhD})^{1}$

${ }^{1}$ Human Health Sciences, Graduate School of Medicine, Kyoto University, Kyoto, Japan ${ }^{2}$ Research Fellow of the Japan Society for the Promotion of Science, Tokyo, Japan ${ }^{3}$ Graduate School of Nursing, Kyoto Prefectural University of Medicine, Kyoto, Japan ${ }^{4}$ Department of Rehabilitation, Hirosaki Stroke Center, Aomori, Japan

Current address correspondence to Yoshihiro Fukumoto,

Faculty of Rehabilitation, Kobe Gakuin University, Kobe, Japan

518 Arise, Ikawadani-cho, Nishi-ku, Kobe 651-2180 Japan.

Phone/Fax: +81-78-974-2461

E-mail: fukumoto@ reha.kobegakuin.ac.jp 


\begin{abstract}
Enhanced echo intensity (EI) on an ultrasound image of skeletal muscle indicates changes in muscle quality, including increases in intramuscular fibrous and adipose tissues. However, it is not known whether muscle quality assessed from the EI of computer-aided gray-scale analysis of an ultrasound image is associated with the muscle strength or body composition of a subject. The objectives of this study were to investigate whether muscle quality assessed from EI measured using gray-scale analysis is associated with muscle strength independently of age or muscle thickness (MT), and to examine the relationship between muscle EI and body composition. Ninety-two healthy women with a mean age of $70.4 \pm 5.5$ years (range, 51-87 years) dwelling in Kyoto, Japan, participated in the study. The MT, subcutaneous fat thickness (FT), and EI of the quadriceps femoris on the right extremity were assessed from transverse ultrasound images. Knee extensor isometric strength was used as a measure of the quadriceps femoris muscle strength. EI was significantly correlated with quadriceps strength independently of age or MT, and stepwise regression analysis revealed that MT and EI were independently associated with quadriceps strength. Importantly, EI showed no significant correlations with FT, percentage of body fat (\%BF), or body mass index (BMI), while FT, BMI, and \%BF did not significantly influence muscle strength. These data suggest that muscle quantity (i.e., MT) and muscle quality assessed from EI measured using computer-aided gray-scale analysis independently contribute to muscle strength in middle-aged and elderly persons.
\end{abstract}

Key words: ultrasound image, muscle echo intensity, muscle thickness, muscle strength, body composition 


\section{Introduction}

Sarcopenia is the loss of muscle mass that results from aging. It is the primary reason for age-related impairments in muscle strength and power (Brooks and Faulkner 1994; Frontera et al. 1991). These impairments include deficits in lower extremity strength, which lead to functional limitations when performing tasks relevant to daily life, such as walking, stair climbing, and chair rising (Arborelius et al. 1992; Bohannon 1997; Rantanen et al. 1994). Therefore, the maintenance of muscle mass during the aging process is critical to ensure that elderly adults can independently carry out the essential activities of daily living.

In addition to age-related reductions in muscle mass (i.e., muscle quantity as determined by muscle volume, cross-sectional area, and thickness), there are changes in muscle quality with aging, including increased adipose tissue accumulation and water content within muscle. Muscle quality can be assessed using computed tomography (CT), which shows a reduced attenuation coefficient due to augmented fat infiltration (Goodpaster et al. 2000). Muscle quality can also be evaluated using the non-invasive, easily accessible, and safe method of ultrasound imaging, whereby an enhanced echo intensity (EI) represents changes caused by increased intramuscular fibrous and adipose tissue (Heckmatt et al. 1982; Pillen et al. 2009; Reimers et al. 1993). Previously, EI has been graded visually, but this approach is subjective and depends on the experience of the observer, and is therefore considered unacceptably inaccurate (Landis and Koch 1977; Pillen et al. 2006). To overcome this limitation, computer-aided gray-scale analysis is being used for quantitative measurement of skeletal muscle EIs (Arts et al. 2010; Bargfrede et al. 1999; Maurits et al. 2003, 2004; Pillen et al. 2006, 2009; Reimers et al 1993; Reimers et al. 1996). Quantitative data generated from computer-aided gray-scale analyses of EIs are more applicable for research purposes because they involve objective evaluations and are suitable for statistical analyses (Pillen et al. 2006). Accordingly, the computerized evaluation of muscle EI has shown higher sensitivity than visual scoring in children suspected of having a neuromuscular disorder (Pillen et al. 2006).

Muscle quality, as well as muscle quantity, is reported to be associated with muscle strength. Previous studies have shown that a reduced attenuation coefficient on CT of mid-thigh and lower leg muscle was a predictor of poor muscle strength and functional limitations independent of muscle cross-sectional area (CSA) (Goodpaster et al. 2001; Sipilä et al. 2004; Visser et al. 2002). These reports suggest that the evaluation of age-related changes in muscle quantity results in an overestimation of the amount of contractile muscle in older persons (Goodpaster et al. 2001; Sipilä et al. 2004; Visser et al. 2002; Yamada et al. 2010). In studies using ultrasound images, Sipilä and Suominen $(1991,1994)$ reported that EI of the quadriceps femoris was significantly correlated with knee extensor strength in older persons. While the results of these ultrasound studies are interesting, they were based on EI values generated by less accurate visual scoring. To verify the correlations identified using visual scoring of ultrasound images, computer-aided gray-scale analyses of EI must be used. Presently, information describing the association of muscle quality assessed from EI measured using gray-scale analysis with 
muscle strength in older persons is limited. It is also unclear whether EI as an index of muscle quality is associated with muscle strength independently of age, and muscle thickness (MT) as an index of muscle quantity. Therefore, the first objective of this study was to investigate whether muscle quality assessed from EI measured using gray-scale analysis is associated with muscle strength independently of age or MT. We hypothesized that EI as an index of muscle quality, as well as MT as an index of muscle quantity, is associated with muscle strength, and that the relationship is not dependent on age or MT.

Evidence suggests there are age-related changes in skeletal muscle composition, such as an increase in intramuscular adipose tissue, and body composition, including increases in visceral fat and subcutaneous fat. Previous study has shown that attenuation of the quadriceps femoris and hamstring muscles on CT were negatively correlated with subcutaneous thigh adipose tissue area, body mass index (BMI), and percentage body fat (\%BF) in older individuals (Goodpaster et al. 2001). However, no study has investigated whether EI determined using ultrasound imaging, which represents the degree of fat infiltration within muscle, is associated with markers of body composition such as \% BF measured using bioelectrical impedance analysis. Therefore, the second objective of this study was to examine the relationship between muscle EI measured using gray-scale analysis and body composition.

\section{Methods}

\section{Participants}

Ninety-two women with a mean age of $70.4 \pm 5.5$ years (range, 51-87), living independently in Kyoto, Japan, participated in this study. All subjects provided written informed consent; the study protocol was approved by the ethics committee of Kyoto University Graduate School and Faculty of Medicine.

Persons were eligible if they were able to walk without assistive devices, reported no history of lower limb trauma or surgery, reported no neuromuscular disorders, reported no acute or chronic diseases that impaired their strength and power, and reported no severe dementia which may have influenced informed consent.

\section{Ultrasound measurement}

Transverse ultrasound images of the quadriceps femoris on the right extremity were obtained with a B-mode ultrasound imaging device (LOGIQ e; GE Healthcare UK Ltd., Chalfont, Buckinghamshire, England) and a multi-frequency linear transducer ( $8-12 \mathrm{MHz})$. Participants were completely relaxed and sitting comfortably with a knee flexion of 60 degrees. During all measurements, an $8-\mathrm{MHz}$ transducer with a 58-dB gain was used. The transducer was positioned perpendicular to the longitudinal axis of the quadriceps femoris at the midpoint between the anterior superior iliac spine and the proximal end of the patella. Two consecutive images were obtained by the same investigator. To ensure the pressure of the transducer was kept to a minimum and that distortion of the skin and subcutaneous tissues caused by excess compression was avoided, a generous amount of contact gel was applied to the skin and real-time 
ultrasonic images were observed.

An electric caliper on frozen transverse images was used to measure: the MT of the quadriceps femoris as the distance between the upper margin of the femoral bone and the lower boundary of the ventral fascia of the rectus femoris (RF), identifying the MT of the quadriceps femoris as the RF and vastus intermedius; and the subcutaneous fat thickness (FT) as the distance between the upper boundary of the ventral fascia and the line separating the dermis from fat (Figure 1). Echo intensities were determined by computer-assisted 8-bit gray-scale analysis using the standard histogram function in Adobe Photoshop Elements (Adobe Systems Inc., San Jose, CA, USA). Regions of interest that included as much of the RF muscles as possible but avoided the surrounding fascia were selected. The mean EI of the regions was expressed as a value between 0 (black) and 255 (white). Mean MT, FT, and EI were calculated using measurements from two images. To assess test-retest reliabilities, intra-class correlation coefficients (ICC1.1) were evaluated using two images taken on two separate days in seven female subjects with a mean age of $60.3 \pm 6.9$ years. ICC (1.1) values were 0.90 for MT, 0.96 for FT, and 0.91 for EI.

\section{Body composition measurement}

\% BF was estimated using segmental bioelectrical impedance analysis, which has been validated for use in the elderly and was conducted as previously described (Yamada et al. 2009). The analysis is based on Ohm's Law and uses impedance indices and a newly developed correction factor for water distribution within the body. It is not influenced by age, sex, or weight (Yamada et al. 2009), and it estimates body composition with a higher accuracy than traditional methods (Ishiguro et al. 2005; Tanaka et al. 2007).

All measurements were taken with the participants in a relaxed supine position on a padded wooden table, arms slightly abducted from the body, forearms pronated, and legs slightly apart. An eight-channel battery-operated impedance instrument (Muscle- $\alpha$; Art Haven 9, Kyoto, Japan) and 12 pre-gelled electrocardiogram tab-type monitoring electrodes $(2 \mathrm{~cm} \times 2 \mathrm{~cm}$, Red Dot, 3M) were used (Yamada et al. 2009). \%BF was calculated using previously validated equations (Yamada et al. 2009).

\section{Muscle strength measurement}

The maximal isometric strength of the knee extensors on the right side was measured using an isometric dynamometer (Isoforce GT-330; OG GIKEN Co., Okayama, Japan). Participants were positioned on the dynamometer in a sitting position with a knee flexion of 60 degrees, the pelvis was fixed using a strap, and a sensor pad was placed $26 \mathrm{~cm}$ distal to the knee joint. After 2-3 practice trials, isometric strength (Newton, N) was measured for $3 \mathrm{~s}$ twice, separated by a 30-s rest period. A maximal value was obtained and torque $(\mathrm{Nm})$ was calculated by multiplying strength $(\mathrm{N})$ by lever arm (m). The ICC $(1.1)$ for the muscle strength measurement was 0.96 . 


\section{Statistical analyses}

Statistical analyses were performed using SPSS (version 17.0; SPSS Japan Inc., Tokyo, Japan). Data are shown as means \pm standard deviations (SD). Correlations between MT, EI, physical characteristics, and muscle strength were calculated using Pearson's correlation coefficients. Partial correlations that examined the effects of age, BMI, and EI on the relationship between MT and muscle strength, and the effects of age and MT on the relationship between EI and muscle strength were evaluated.

Stepwise regression analysis was performed to determine the relative importance of MT and EI in muscle strength. In addition to MT and EI, independent variables that showed significant correlations with muscle strength by Pearson's correlation coefficients were used as confounding factors. To monitor for a multicollinearity effect, the variance inflation factor (VIF) was calculated. A model was considered ill conditioned if the VIF was higher than 10 (Kellis et al. 2000). Statistical significance was defined as $P$ $<0.05$.

\section{Results}

Table 1 shows the physical characteristics, ultrasound measurements, and muscle strength of the participants.

Table 2 shows the correlation coefficients between MT, EI, the physical characteristics, and the muscle strength of the participants. MT showed a significant negative correlation with age, and a significant positive correlation with BMI and muscle strength. MT was not significantly associated with \%BF or FT. EI showed a significant positive correlation with age, and significant negative correlations with MT and muscle strength. EI was not significantly associated with BMI, \%BF, or FT. Muscle strength was significantly correlated with age and BMI.

Table 3 shows the partial correlation coefficients between EI, MT, and muscle strength. With age as a control variable, both MT and EI were significantly correlated with muscle strength. With age, BMI, and EI as control variables, MT showed a significant positive correlation with muscle strength. With age and MT as control variables, EI showed a significant negative correlation with muscle strength.

Stepwise regression analyzes identified MT and EI, but not age and BMI, as independent variables significantly associated with muscle strength (Table 4). The VIFs ranged from 1.177 to 1.267.

\section{Discussion}

Previous reports indicated that muscle quality estimated from visual scoring of EI or attenuation coefficients determined by CT was associated with muscle strength and physical performance (Sipilä and Suominen 1991, 1994; Goodpaster et al. 2001; Sipilä et al. 2004; Visser et al. 2002). However, no studies have investigated whether muscle quality assessed from EI measured using gray-scale analysis of ultrasound images is associated with muscle strength or body composition. The present study was the first to investigate the relationships between EI measured using gray-scale analysis with muscle strength and 
body composition in middle-aged and elderly women.

Evidence suggests that there is an increase in adipose tissue accumulation and a reduction in muscle mass within skeletal muscle with aging. Previous studies have reported an age-related increase in the EI of the upper and lower extremity muscles in healthy individuals, probably due to the replacement of contractile tissue by fat and fiber (Arts et al. 2010; Reimers et al. 1996), and CT showed that the attenuation of the quadriceps femoris and hamstring muscles is decreased in the elderly (70-80 yrs) (Goodpaster et al. 2001). In accordance with these results, we observed an age-related increase in EI and a decrease in MT of the quadriceps femoris muscle. Taken together, these data suggest that the loss of muscle contractile tissue with aging is larger than the decrease in muscle size.

In our study, EI was negatively correlated with muscle strength. As age was also correlated with muscle strength, we performed partial correlation analysis with age as a control variable and found that EI was still significantly correlated with muscle strength. Furthermore, when MT was used as a covariate, the result was the same as that obtained with Pearson's correlation. Thus, the association between muscle quality assessed from EI and muscle strength was not influenced by age or MT. Stepwise regression analyses showed that the MT and EI of the quadriceps femoris were significantly and independently associated with muscle strength. These data suggest that muscle quantity (i.e., muscle size) and muscle quality (i.e., the amounts of fibrous and adipose tissue within the muscle) independently contribute to muscle strength. Sarcopenia, the age-related decrease in muscle size, was reported to involve reduction or dysfunction of muscle satellite cells (Hawke and Garry 2001; Machida and Booth 2004). On the other hand, recent study has demonstrated that the amount of adipose tissue within muscles is affected not by satellite cells but by mesenchymal stem cells (Uezumi et al. 2010). The results obtained in our study, that muscle quality is associated with muscle strength independently of muscle quantity, could involve the mesenchymal stem cells rather than satellite cells.

The relative increase in muscle EI (i.e., the reduction of muscle quality) with aging may result in an age-related decrease in specific force (force production per unit muscle size). However, in our study, although statistically significant, the influence of muscle quality assessed from EI on muscle strength was relatively low, accounting for $16 \%$ of the variance. These data suggest that age-related decreases in muscle strength may be associated with factors other than muscle quality, including a reduced proportion of fast type II fibers (Larsson 1997; Sipilä et al. 2004) and decreased neural activation of the agonist muscle (Stevens et al. 2001). In addition, coactivation (the opposing mechanical action of the antagonist muscle) is reported to be higher in elderly adults and may contribute to reduced force production (Izquierdo et al. 1999; Macaluso et al. 2002).

A further important finding in our study was that the EI of the quadriceps femoris was not correlated with the FT of the anterior thigh, \%BF, or BMI. These results suggest that the use of FT, \%BF, and BMI as indices of subcutaneous fat, visceral fat, and obesity are not appropriate indicators of adipose and fibrous tissues within individual skeletal muscles and that, despite a small \%BF, skeletal muscle may 
contain a substantial amount of fat. In addition, FT, BMI, and \%BF did not significantly influence muscle strength, suggesting that these parameters should not be used as indices of muscle strength. Instead, our data indicate that the EI of a particular muscle, which represents the intramuscular fibrous and adipose tissue, will provide a more accurate index of the strength of that muscle. Furthermore, because the combination of lower skeletal mass and higher \%BF, defined as sarcopenic obesity, was reported to be more strongly associated with disability in elderly persons than either body composition type alone (Baumgartner et al. 2004), it is possible that the combination of decreased muscle mass and increased fat tissue within a specific skeletal muscle has a greater influence on the functional limitations of that muscle than each parameter alone; future studies are needed to clarify this proposal. In contrast to our data, CT showed that attenuation of the quadriceps femoris and hamstring muscles were negatively correlated with subcutaneous thigh adipose tissue area, BMI, and \%BF in older individuals (Goodpaster et al. 2001). The latter study consisted of a large cohort of white and black males and females, which may have accounted for the disparate observations.

Our study has some limitations. First, this study only investigated the influence of muscle quality assessed from EI on the muscle strength of the quadriceps femoris. However, studies in patients with non-dystrophic myotonia, showed that the EIs of the biceps brachii and tibialis anterior were not correlated with muscle strength, although the EI of the forearm flexors was significantly correlated with grip strength (Trip et al. 2009). This indicates that the influence of muscle quality assessed from EI on strength may differ between muscles. Therefore, our results may not be applicable to other skeletal muscles in humans. Second, our cross-sectional data does not allow us to identify a causal relationship between adipose and fibrous tissue in muscle, and changes in the muscle strength of the participants. Longitudinal studies are needed to further investigate these associations.

In conclusion, we found that muscle quality assessed from EI of skeletal muscle was related to muscle strength independently of age or MT, and that EI was not associated with \%BF, BMI, or FT. We propose that ultrasound is a low-cost rapid method suitable for the assessment of EI as an index of muscle quality, which is independently associated with muscle strength of middle-aged and elderly women. Furthermore, because it is portable, it is convenient for people living in nursing homes or who have restricted mobility within their own communities and limited access to health centres and hospitals. Our data suggest that muscle quantity (i.e., muscle size) and muscle quality assessed from EI measured using computer-aided gray-scale analysis independently contribute to muscle strength in middle-aged and elderly persons.

\section{Acknowledgments}

The authors would like to thank Megumi Nakamura, Saori Shibuta, Shuhei Mori, Yui Takagi, and Mitsuhiro Masaki (Human Health Sciences, Graduate School of Medicine, Kyoto University) for their practical and technical assistance. The authors also thank all of the individuals who participated in the 
study. This study was not funded by any institutions, agencies, or companies.

\section{Conflicts of interest}

The authors have no conflicts of interest to disclose.

\section{References}

Arborelius UP, Wretenberg P, Lindberg F (1992) The effects of armrests and high seat heights on lower-limb joint load and muscular activity during sitting and rising. Ergonomics 35(11): 1377-1391

Arts IM, Pillen S, Schelhaas HJ, Overeem S, Zwarts MJ (2010) Normal values for quantitative muscle ultrasonography in adults. Muscle Nerve 41:32-41. doi: 10.1002/mus.21458

Bargfrede M, Schwennicke A, Tumani H, Reimers CD (1999) Quantitative ultrasonograpy in focal neuropathies as compared to clinical and EMG findings. Eur $\mathrm{J}$ Ultrasound 10:21-29. doi:10.1016/S0929-8266(99)00040-3

Baumgartner RN, Wayne SJ, Waters DL, Janssen I, Gallagher D, Morley JE (2004) Sarcopenic obesity predicts instrumental activities of daily living disability in the elderly. Obes Res 12(12):1995-2004. doi: 10.1038/oby.2004.250

Bohannon RW (1997) Comfortable and maximum walking speed of adults aged 20-79 years: reference values and determinants. Age and Aging 26(1): 15-19. doi: 10.1093/ageing/26.1.15

Brooks SV, Faulkner JA (1994) Skeletal muscle weakness in old age: underlying mechanisms. Med Sci Sports Exerc 26(4):432-439.

Frontera WR, Hughes VA, Lutz KJ, Evans WJ (1991) A cross-sectional study of muscle strength and mass in 45- to 78-yr-old men and women. J Appl Physiol. 71(2):644-650

Goodpaster BH, Carlson CL, Visser M, et al. (2001) Attenuation of skeletal muscle and strength in the elderly: The Health ABC Study. J Appl Physiol. 90(6):2157-2165

Goodpaster BH, Kelley DE, Thaete FL, He J, Ross R (2000) Skeletal muscle attenuation determined by computed tomography is associated with skeletal muscle lipid content. J Appl Physiol. 89(1):104-110

Hawke TJ, Garry DJ (2001) Myogenic satellite cells: physiology to molecular biology. J Appl Physiol 91(2):534-551.

Heckmatt JZ, Leeman S, Dubowitz V (1982) Ultrasound imaging in the diagnosis of muscle disease. J Pediatr 101: 656-660.

Ishiguro N, Kanehisa H, Miyatani M, Masuo Y, Fukunaga T (2005) A comparison of three bioelectrical impedance analyses for predicting lean body mass in a population with a large difference in muscularity. Eur J Appl Physiol 94:25-35. doi: 10.1007/s00421-004-1259-2

Izquierdo M, Ibañez J, Gorostiaga E, et al. (1999) Maximal strength and power characteristics in isometric and dynamic actions of the upper and lower extremities in middle-aged and older men. Acta Physiol Scand 167(1):57-68. doi: 10.1046/j.1365-201x.1999.00590.x 
Kellis S, Kellis E, Manou V, Gerodimos V (2000) Prediction of knee extensor and flexor isokinetic strength in young male soccer players. J Orthop Sports Phys Ther 30(11): 693-701.

Landis JR, Koch GG (1977) The measurement of observer agreement for categorical data. Biometrics 33(1):159-174.

Larsson L, Li X, Frontera WR (1997) Effects of aging on shortening velocity and myosin isoform composition in single human skeletal muscle cells. Am J Physiol 272(2 Pt 1):C638-49.

Macaluso A, Nimmo MA, Foster JE, Cockburn M, McMillan NC, De Vito G (2002) Contractile muscle volume and agonist-antagonist coactivation account for differences in torque between young and older women. Muscle Nerve 25(6):858-863. doi: 10.1002/mus.10113

Machida S, Booth FW (2004) Increased nuclear proteins in muscle satellite cells in aged animals as compared to young growing animals. Exp Gerontol 39(10):1521-1525. doi: 10.1016/j.exger.2004.08.009

Maurits NM, Beenakker EA, van Schaik DE, Fock JM, van der Hoeven JH (2004) Muscle ultrasound in children: normal values and application to neuromuscular disorders. Ultrasound Med Biol 30:1017-1027. doi: 10.1016/j.ultrasmedbio.2004.05.013

Maurits NM, Bollen AE, Windhausen A, De Jager AE, Van Der, Hoeven JH (2003) Muscle ultrasound analysis: normal values and differentiation between myopathies and neuropathies. Ultrasound Med Biol 29:215-225. doi: 10.1016/S0301-5629(02)00758-5

Pillen S, Tak RO, Zwarts MJ, et al (2009) Skeletal muscle ultrasound: correlation between fibrous tissue and echo intensity. Ultrasound Med Biol 35: 443-446. doi: 10.1016/j.ultrasmedbio.2008.09.016

Pillen S, van Keimpema M, Nievelstein RA, Verrips A, Kruijsbergen-Raijmann W, Zwarts MJ (2006) Skeletal muscle ultrasonography: visual versus quantitative evaluation. Ultrasound Med Biol 32:1315-1321. doi: 10.1016/j.ultrasmedbio.2006.05.028

Rantanen T, Era P, Heikkinen E (1994) Maximal isometric strength and mobility among 75-year-old men and women. Age and Aging 23(2): 132-137. doi: 10.1093/ageing/23.2.132

Reimers CD, Schlotter B, Eicke BM, Witt TN (1996) Calf enlargement in neuromuscular diseases: a quantitative ultrasound study in 350 patients and review of the literature. J Neurol Sci 143: 46 -56. doi: $10.1016 / \mathrm{S} 0022-510 \mathrm{X}(96) 00037-8$

Reimers K, Reimers CD, Wagner S, Paetzke I, Pongratz DE (1993) Skeletal muscle sonograpy: a correlative study of echogenicity and morphology. J Ultrasound Med 12:73-77.

Sipilä S, Koskinen SO, Taaffe DR, et al. (2004) Determinants of lower-body muscle power in early postmenopausal women. J Am Geriatr Soc 52(6):939-944. doi: 10.1111/j.1532-5415.2004.52261.x

Sipilä S, Suominen H (1991) Ultrasound imaging of the quadriceps muscle in elderly athletes and untrained men. Muscle Nerve 14(6):527-533. doi: 10.1002/mus.880140607

Sipilä S, Suominen H (1994) Knee extension strength and walking speed in relation to quadriceps muscle composition and training in elderly women. Clin Physiol 14(4):433-442. 
Stevens JE, Binder-Macleod S, Snyder-Mackler L (2001) Characterization of the human quadriceps muscle in active elders. Arch Phys Med Rehabil 82(7):973-978. doi: 10.1053/apmr.2001.23995

Tanaka NI, Miyatani M, Masuo Y, Fukunaga T, Kanehisa H (2007) Applicability of a segmental bioelectrical impedance analysis for predicting the whole body skeletal muscle volume. J Appl Physiol 103:1688-1695. doi: 10.1152/japplphysiol.00255.2007

Trip J, Pillen S, Faber CG, van Engelen BG, Zwarts MJ, Drost G. (2009) Muscle ultrasound measurements and functional muscle parameters in non-dystrophic myotonias suggest structural muscle changes. Neuromuscul Disord 19: 462-467. doi: 10.1016/j.nmd.2009.06.369

Uezumi A, Fukada S, Yamamoto N, Takeda S, Tsuchida K. (2010) Mesenchymal progenitors distinct from satellite cells contribute to ectopic fat cell formation in skeletal muscle. Nat Cell Biol. 12(2):143-152. doi: 10.1038/ncb2014

Visser M, Kritchevsky SB, Goodpaster BH, Newman AB, Nevitt M, Stamm E, Harris TB (2002) Leg muscle mass and composition in relation to lower extremity performance in men and women aged 70 to 79: the health, aging and body composition study. J Am Geriatr Soc 50(5):897-904. doi: 10.1046/j.1532-5415.2002.50217.x

Yamada Y, Masuo Y, Yokoyama K, et al. (2009) Proximal electrode placement improves the estimation of body composition in obese and lean elderly during segmental bioelectrical impedance analysis. Eur $\mathrm{J}$ Appl Physiol. 107(2):135-144. doi: 10.1007/s00421-009-1106-6

Yamada Y, Schoeller DA, Nakamura E, Morimoto T, Kimura M, Oda S (2010) Extracellular water may mask actual muscle atrophy during aging. J Gerontol A Biol Sci Med Sci 65(5):510-516. doi: 10.1093/gerona/glq001 
Table 1. Physical characteristics, ultrasound measurements, and the muscle strength of the participants (n $=92)$

\begin{tabular}{|c|c|c|}
\hline & Mean \pm SD & Range \\
\hline \multicolumn{3}{|l|}{ Physical characteristics } \\
\hline Age (years) & $70.4 \pm 6.6$ & $51-87$ \\
\hline Height $(\mathrm{cm})$ & $151.1 \pm 5.4$ & $141.2-173.3$ \\
\hline Weight (kg) & $50.4 \pm 6.2$ & $37.0-68.9$ \\
\hline BMI $\left(\mathrm{kg} / \mathrm{m}^{2}\right)$ & $22.0 \pm 2.3$ & $16.2-29.9$ \\
\hline$\% \mathrm{BF}$ & $34.7 \pm 6.3$ & $20.1-51.0$ \\
\hline \multicolumn{3}{|c|}{ Ultrasound measurements } \\
\hline FT $(\mathrm{cm})$ & $1.11 \pm 0.38$ & $0.37-2.22$ \\
\hline MT (cm) & $3.57 \pm 0.51$ & $2.14-4.82$ \\
\hline EI & $98.8 \pm 10.0$ & $75.3-129.9$ \\
\hline Muscle strength (Nm) & $87.3 \pm 26.8$ & $33.8-156.8$ \\
\hline
\end{tabular}

BMI: body mass index; \%BF: percentage body fat; FT: subcutaneous fat thickness; MT: muscle thickness; EI: echo intensity. 
Table 2. Correlation coefficients between MT, EI, the physical characteristics, and the muscle strength of the participants $(n=92)$

\begin{tabular}{lccccccc}
\hline & MT & EI & Age & BMI & \% BF & FT & Muscle strength \\
& & & & & & \\
\hline MT & - & $-0.33 * *$ & $-0.40^{* *}$ & $0.39 * *$ & 0.11 & 0.14 & $0.47 * *$ \\
EI & & - & $0.34 * *$ & -0.12 & 0.08 & -0.19 & $-0.40^{* *}$ \\
Age & & - & -0.13 & -0.05 & -0.17 & $-0.32^{* *}$ \\
BMI & & & - & $0.74 * *$ & $0.61 * *$ & $0.22^{*}$ \\
\%BF & & & & - & $0.57 * *$ & -0.04 \\
FT & & & & & - & 0.15 \\
Muscle strength & & & & & & - \\
\hline
\end{tabular}

MT: muscle thickness; EI: echo intensity; BMI: body mass index; \%BF: percentage body fat; FT: subcutaneous fat thickness.

Statistical significance: $* P<0.05, * * P<0.01$ 
Table 3. Partial correlation coefficients between MT, EI, and muscle strength $(n=92)$

\begin{tabular}{clc}
\hline & Control variables & $\begin{array}{c}\text { Partial correlation coefficients } \\
\text { with the muscle strength }\end{array}$ \\
\hline MT & Age & $0.40^{* *}$ \\
& Age, BMI, and EI & $0.32^{* *}$ \\
\hline EI & Age & $-0.33^{* *}$ \\
& Age and MT & $-0.26^{*}$ \\
\hline
\end{tabular}

MT: muscle thickness; EI: echo intensity; BMI: body mass index.

Statistical significance: $* P<0.05, * * P<0.01$ 
Table 4. Factors associated with muscle strength on stepwise regression analysis $(n=92)$

\begin{tabular}{|c|c|c|c|c|c|c|c|}
\hline \multirow{2}{*}{$\begin{array}{l}\text { Dependent } \\
\text { variables }\end{array}$} & \multirow{2}{*}{$\begin{array}{l}\text { Independent } \\
\text { Variables }\end{array}$} & \multirow[b]{2}{*}{ Coefficient } & \multirow{2}{*}{$\begin{array}{c}\text { Standardized } \\
\text { Coefficient }\end{array}$} & \multirow[b]{2}{*}{$\mathrm{t}$ value } & \multirow[b]{2}{*}{$p$ value } & \multicolumn{2}{|c|}{$95 \%$ Confidence interva } \\
\hline & & & & & & lower & upper \\
\hline Muscle strength & MT & 20.3 & 0.38 & 4.05 & $<0.01$ & 10.3 & 30.2 \\
\hline$(\mathrm{Nm})$ & EI & -0.73 & -0.27 & -2.88 & $<0.01$ & -1.24 & -0.23 \\
\hline $\mathrm{R}^{2}=0.29$ & & & & & & & \\
\hline
\end{tabular}

MT: muscle thickness; EI: echo intensity 


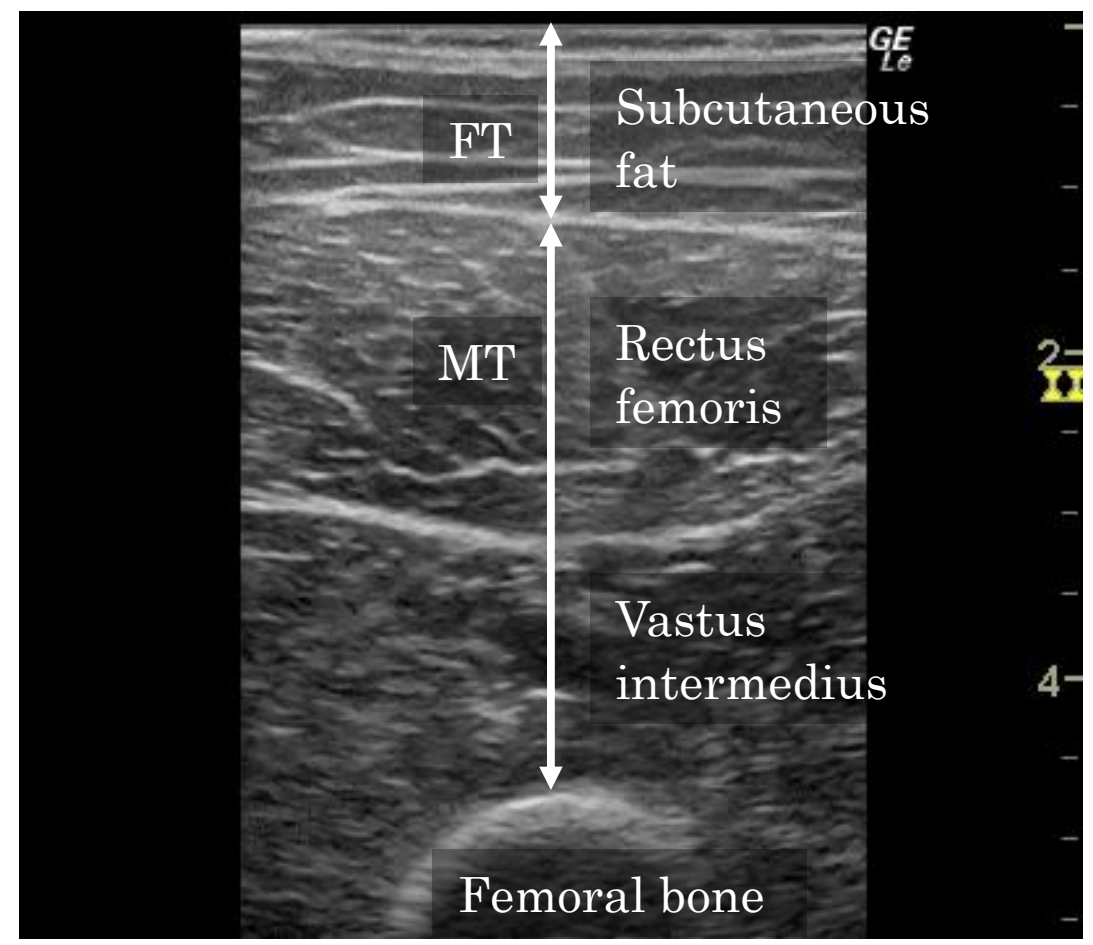

Figure 1. Measurement of muscle thickness (MT) and subcutaneous fat thickness (FT). 